\title{
Preliminary Thermal Analysis of Critical Components in ITER Lower Port \#02*)
}

\author{
Toshiyuki MARUYAMA ${ }^{1,2)}$, Sin-iti KITAZAWA ${ }^{1)}$, Hiroaki OGAWA ${ }^{1)}$ and Kiyoshi ITAMI ${ }^{1)}$ \\ 1) Naka Fusion Institute, Japan Atomic Energy Agency, Naka, Ibaraki 311-0193, Japan \\ ${ }^{2)}$ TOYAMA Co. Ltd., Yamakita-machi, Ashigarakami-gun, Kanagawa 258-0112, Japan
}

(Received 27 November 2015 / Accepted 5 April 2016)

\begin{abstract}
ITER diagnostic lower ports will be used to exchange divertor cassettes and install diagnostic systems. Invessel components of diagnostic lower port \#02 (LP\#02) will be mounted on a divertor cassette and a support structure, so-called a diagnostic rack, to be removed and reinstalled collectively. Japan Domestic Agency is responsible for procurements of an integration engineering of LP\#02 and a divertor impurity monitor, a main tenant system in LP\#02. This paper presents preliminary thermal analyses for two critical components in LP\#02, the diagnostic rack and a mirror box on the divertor cassette, which will be exposed to the harsh thermal load from plasma. Realistic fabrication methods for cooling the diagnostic rack and a required cooling path layout of the mirror box on the divertor cassette have been assessed to implement the preliminary design from the viewpoint of thermal analysis.
\end{abstract}

(C) 2016 The Japan Society of Plasma Science and Nuclear Fusion Research

Keywords: ITER, JADA, thermal analysis, lower port, diagnostics

DOI: $10.1585 /$ pfr. 11.2405083

\section{Introduction}

The diagnostic lower ports will be used to exchange divertor cassettes and install diagnostic systems via remote handling $(\mathrm{RH})$ [1]. Some diagnostics will be assembled in the divertor cassettes, whereas others will be inserted in the lower ports after installation of the divertor cassettes to measure the plasmas from the bottom of the ITER vacuum vessel (VV). Therefore, the diagnostic systems in the lower ports should be designed to be removed and reinstalled. In-VV diagnostic components of the lower port will be mounted on a divertor cassette and a support structure, so-called a diagnostic rack, to be removed and reinstalled as a unit [2,3].

Japan Domestic Agency (JADA) is responsible for integrating engineering of diagnostic lower port \#02 (LP\#02) because JADA is also responsible for procuring the ITER divertor impurity monitor (DIM), a main tenant system in LP\#02 [4]. JADA will manufacture the diagnostic rack and DIM components. Procurement arrangements (PA) of LP\#02 and DIM are separated. Currently, the PA documents for LP\#02 to be agreed between JADA and the ITER Organization are being prepared. The PA of DIM was signed on August 2013 and the preliminary design is currently progressing.

In this paper, we performed the preliminary thermal analyses for two critical components in LP\#02, the diagnostic rack and the mirror box of DIM on the divertor cassette, because these components will be installed near the

author's e-mail: maruyama.toshiyuki@qst.go.jp

*) This article is based on the presentation at the 25th International Toki Conference (ITC25). plasma and exposed to the harsh thermal load from plasma.

\section{Preliminary Thermal Analyses for Cooling Structures of Diagnostic Rack}

\subsection{Background}

There are a few pending issues from conceptual design of generic lower port rack [3], which was closed on February 2015. In our previous work [2], we found that the diagnostic rack needs water cooling to withstand nuclear heating loads. In particular, the plasma-facing front end of the rack receives the high heat load and it must be cooled intensively. In this paper, we focus on a water-cooling structure of the rack. Choice of a method for fabricating a water-cooling system in the rack is very important from the viewpoint of the whole structure and its manufacturability, because there are several disadvantages and limitations in each methods. Therefore, it is necessary to assess available fabrication methods and cooling path layouts that can withstand the assumed heat load.

The drilling fabrication method and the joining pipe method are realistic candidate fabrication methods. In the case of the drilling method, high cooling performance can be obtained because thermal contact between the structure and cooling path is complete. However, open holes on the structure due to drilling must be sealed by welding. These welding sealed portions should act as a vacuum boundary, and $100 \%$ volumetric nondestructive testing (NDT) is required according to the ITER project specifications. It is difficult to perform NDT of the inside cooling path. 
In addition, it is difficult to fabricate the complex cooling path layout by means of drilling. Conversely, the joining pipe method ensures easy fabrication and considerable reduction in welded area on the vacuum boundary. However, the cooling performance on the joining pipe method is lower because thermal contact between the structure and the cooling pipes is not complete.

\subsection{Conditions}

We assessed the cooling performance of the drilling method and joining pipe method by finite element method (FEM) analysis using ANSYS 15.0. Since the rack is a structure of the plate configuration, we used simplified analysis models of the rack front end to assess cooling performance by extracting the highest heat load area. The model is a simple plate structure with the same size as the rack front end (width $800 \mathrm{~mm}$, depth $400 \mathrm{~mm}$, and thickness $40 \mathrm{~mm}$ ), and the model is considered as the cooling path for each fabrication method. We analyzed two models, shown in Figs. 1 and 2. In model of joining pipe method, the contact area between the pipe and the plate structure is $15 \%$ of the entire outer surface area of the pipe, which we assumed as the minimum value (worst case) in the case that the pipes were welded directly to the flat surface. The material used in the models is austenitic stainless steel SS316L(N)-IG. The inner diameter of the cooling path is $19 \mathrm{~mm}$. The cooling water flow rate is $20 \mathrm{l} / \mathrm{min}$ at a temperature of $70^{\circ} \mathrm{C}$ and under a hydraulic pressure of $4 \mathrm{MPa}$.

The thermal load conditions for nuclear heating that have been estimated in the lower ports are used [3]. The model is divided into three parts, and different thermal load values are applied to these parts as follows: $0.2 \mathrm{MW} / \mathrm{m}^{3}$ to the central part, and $0.1 \mathrm{MW} / \mathrm{m}^{3}$ to the other parts (see
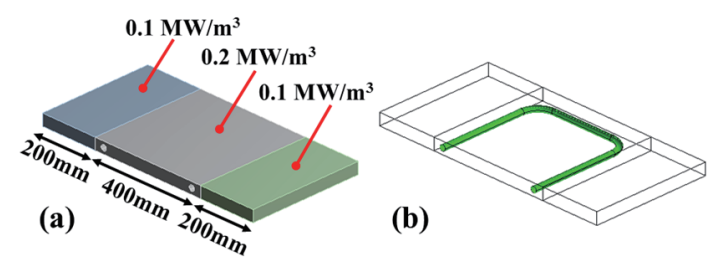

Fig. 1 Analysis model of drilling method: (a) overview and (b) cooling path layout.

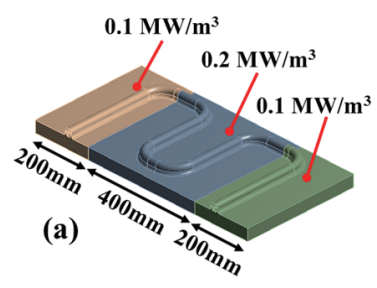

(b)

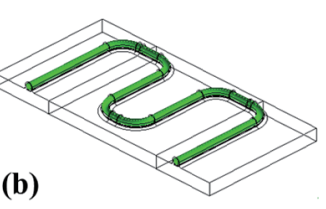

Fig. 2 Analysis model of joining pipe method: (a) overview and (b) cooling path layout.
Figs. 1 and 2). In this analysis, a transient thermal scenario on ITER plasma operation is employed and pulses of $450 \mathrm{~s}$ are generated with a repetition time of $1800 \mathrm{~s}$. Since the diagnostic rack is hidden behind the divertor cassettes and is therefore not exposed directly to the radiation heat fluxes from the divertor plasmas, it is not taken into account in the study.

\subsection{Results}

The analyzed temperature and stress distributions are shown in Figs. 3 and 4. In the both cases, the stress was lower than the allowable stress of SS316L(N)-IG (193 MPa at $150{ }^{\circ} \mathrm{C}$, from SDC-IC [5]). The drilling method can be used from the viewpoint of manufacturability if the cooling path layout is simple and short.

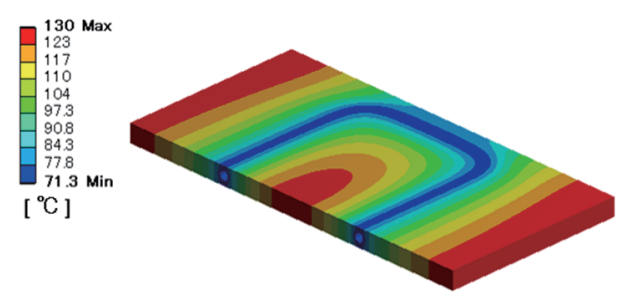

(a)

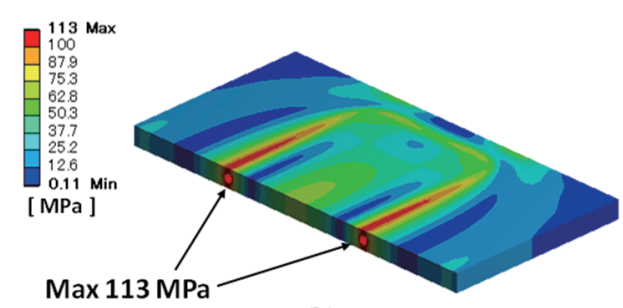

(b)

Fig. 3 Temperature and stress distributions of drilling method model: (a) temperature and (b) equivalent stress.

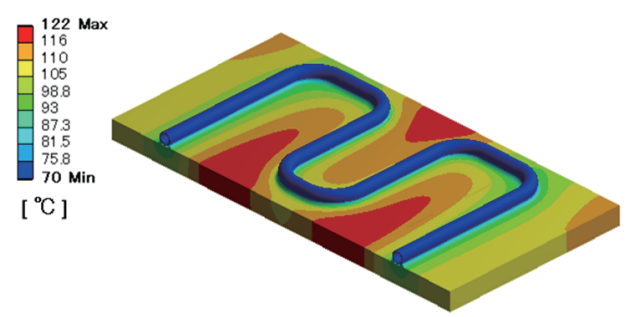

(a)

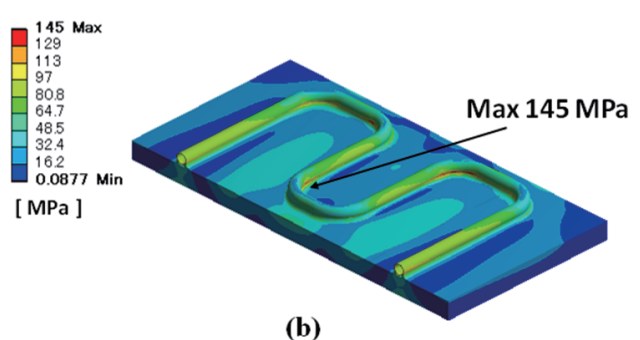

Fig. 4 Temperature and stress distributions of joining pipe method model: (a) temperature and (b) equivalent stress. 
On the other hand, the joining pipe method needs a longer cooling path than the drilling method. The maximum stress of the joining pipe model is higher than that of the drilling model, although the maximum temperature of the former is lower than that of the latter. With the joining pipe method, the maximum stress is generated on the welded part at the corner of the pipe. Therefore, stress concentration can occur easily in the welded areas. In the joining pipe method, it is necessary to ensure adequate contact area between the structure and the cooling pipes. For example, if the grooves are fabricated to fit the pipes before joining, we expect that it would be easy to maintain adequate contact area, that is, over $15 \%$.

Both methods are available for the rack from the viewpoint of thermal analysis. The detailed structure of the rack shall be designed by consulting on this result to implement manufacturability. To verify integrity of the rack, further structural analyses of other loads (electromagnetic force, seismic load, etc.) should be executed.

\section{Preliminary Thermal Analyses for Mirror Box on the Divertor Cas- sette}

\subsection{Background}

The DIM is a diagnostic system for observing light from the fusion plasma near the divertor. The measurement is performed by a conventional method that identifies hydrogen and impurity species from peaks by a spectroscopic method. For this purpose, a mirror box will be attached on the divertor cassette, shown in Fig. 5. The mirror box has functions, to mount mirrors, to shield light and to protect the mirrors against the particle bombardment, where the thermal load is highest on the cassette mirror box compared with those of the upper, equatorial and lower port. We performed preliminary thermal analysis of the mirror box and assessed the required cooling path layout to withstand those thermal loads.

\subsection{Conditions}

We performed the thermal analysis by FEM analysis using ANSYS 15.0. The preliminary optical design for DIM has been performed [6]. According to this design, the mechanical design of the mirror box was developed. The analysis model of mirror box is shown in Fig. 6. The mirrors are mounted inside of the mirror box, and the cooling cover box has function to protect these mirrors. The material used in the models is austenitic stainless steel SS316L(N)-IG. The cooling water is at a temperature of $70{ }^{\circ} \mathrm{C}$ and under a hydraulic pressure of $4 \mathrm{MPa}$. The inner diameter of the cooling path and water flow rate in plasma facing parts were $10 \mathrm{~mm}$ diameter and $10 \mathrm{l} / \mathrm{min}$, and those in others were $8 \mathrm{~mm}$ diameter and $3 \mathrm{l} / \mathrm{min}$. The thermal load conditions for nuclear heating and radiation heat flux in the divertor area were referred $[7,8]$. The nuclear heating is $2 \mathrm{MW} / \mathrm{m}^{3}$ to $1 \mathrm{MW} / \mathrm{m}^{3}$. The radiation heat flux is

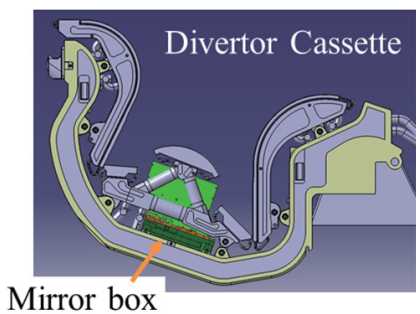

Fig. 5 Schematic view of mirror box on divertor cassette.

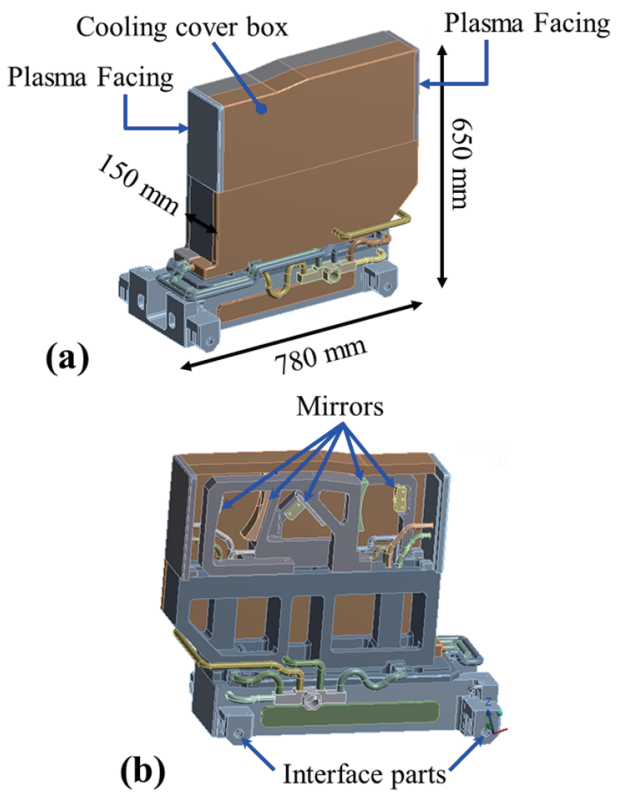

Fig. 6 Analysis model of mirror box: (a) overview and (b) inside box view.

$0.15 \mathrm{MW} / \mathrm{m}^{2}$ to $0.02 \mathrm{MW} / \mathrm{m}^{2}$. The thermal loads of the cover box are mainly caused by the radiation for a structure of the plate configuration. The transient thermal scenario, the same as the thermal analyses for the rack, is employed in this analysis. The temperature on the interface parts of mirror box was assumed to be $100{ }^{\circ} \mathrm{C}$ as the temperature of the divertor.

\subsection{Results}

The resultant cooling path layout is shown in Fig. 7, and the analyzed temperature and stress distributions are shown in Fig. 8. The mirror box, in particular the cooling cover box, needs a lot of cooling paths to withstand the thermal loads. Since the load of radiation heat flux on the cover box is extremely high, the distance between the cooling paths and outer surface of the cover box which receives radiation heat flux load was set to $<\sim 4 \mathrm{~mm}$ in this model in order to keep tolerance of stress.

It is challenging to manufacture the labyrinthine cooling path of the cover box by milling and welded sealing. On the other hand, the cooling path of other mirror box parts will be manufactured by the composite of some method (joining pipes, drilling, milling \& welding). 


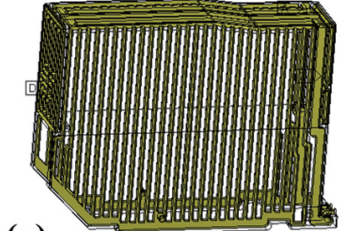

(a)

(b)

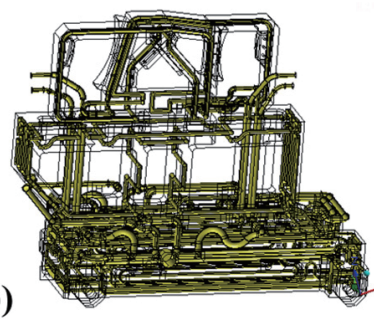

Fig. 7 Cooling path layout of mirror box: (a) cooling cover box and (b) other parts.

(a)

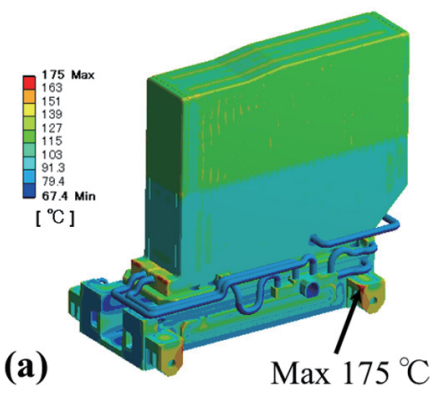

(b)

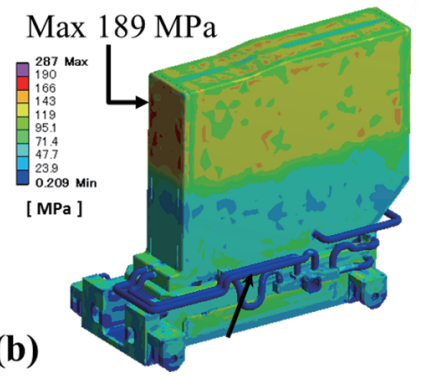

Fig. 8 Temperature and stress distributions of mirror box: (a) temperature and (b) equivalent stress.

Plenty of water is needed for cooling the whole of mirror box (total $\sim 100 \mathrm{~L} / \mathrm{min}$ in the current assumption), but the water supply is capped. The allowable value of water supply for the mirror box has not yet been defined, but the total amount to be distributed to a divertor cassette including the mirror box is $1000 \mathrm{~L} / \mathrm{min}$. Therefore, the cooling path layout will be resolved to keep the required flow rate in the detailed design.

In addition, the stress on the plasma facing parts is close to the tolerance. The combination with electromag- netic force, seismic load and others will also be resolved in the detailed design.

\section{Summary}

JADA will procure the integrating engineering and the components of LP\#02. The diagnostic rack and the mirror box on the divertor cassette are critical components for the LP\#02 engineering, and these components have to withstand the harsh thermal loads. We performed the preliminary thermal analyses. For the diagnostic rack, the cooling structure was analyzed by using simplified models, and then we found that both drilling method and joining pipe method are available for fabricating the cooling path of the rack from the thermal analysis. The detailed structure of the rack shall be designed by consulting on this result to implement manufacturability. To verify integrity of the rack, further structural analyses of other loads (electromagnetic force, seismic load, etc.) should be executed. For the mirror box, the preliminary design of cooling path layout was developed from the thermal analysis, and then we found that the mirror box needs the labyrinthine cooling path and a thickness of $<\sim 4 \mathrm{~mm}$ on outer surface of the cover box which receives highest radiation heat flux. The detailed design of cooling path layout to keep the required flow rate and the analyses of combination loads will be performed.

\section{Acknowledgements}

The authors are grateful to T. Ono (JAEA) and Dr. K. Ioki (Toyama Co., ltd.) for their fruitful discussions, and to K. Ohmori (Nikon Corporation) for supplying CAD model of the mirror box.

\section{Disclaimer}

The views and opinions expressed herein do not necessarily reflect those of the ITER Organization.

[1] B. Levesy et al., Fusion Eng. Des. 86, 1812 (2011).

[2] S. Kitazawa, T. Maruyama, H. Ogawa, K Itami and N. Casal, Plasma Fusion Res. 10, 3402044 (2015).

[3] N. Casal et al., Fusion Eng. Des. 96-97, 83 (2015).

[4] H. Ogawa, T Sugie, S Kasai, A Katsunuma, H Hara, N. Takeyama and Y. Kusama, Fusion Eng. Des. 83, 1405 (2008).

[5] Structural Design Criteria For ITER In-Vessel Components (SDC-IC), G 74 MA 8 01-05-28 W 0.2 (2012).

[6] S. Kitazawa, H. Ogawa, A. Katsunuma, D, Kitazawa and K. Ohmori, Fusion Eng. Des. 101, 209 (2015).

[7] R. Villari et al., Fusion Eng. Des. 88, 2006 (2013).

[8] A. Encheva et al., Fusion Eng. Des. 86, 1323 (2011). 